\title{
TOEPLITZ OPERATORS WITH QUASI-HOMOGENEUOS QUASI-RADIAL SYMBOLS ON SOME WEAKLY PSEUDOCONVEX DOMAINS
}

\author{
RAUL QUIROGA-BARRANCO AND ARMANDO SANCHEZ-NUNGARAY
}

\begin{abstract}
On the weakly pseudo-convex domains $\Omega_{p}^{n}$ we introduce quasihomogeneous quasi-radial symbols. These are used to prove the existence of a commutative Banach algebra of Toeplitz operators on Bergman space of $\Omega_{p}^{n}$. We also show that group theoretic and geometric properties for our symbols are satisfied. The results presented here contain the geometric description of the symbols introduced by N. Vasilevski in 12 for the unit ball $\mathbb{B}^{n}$.
\end{abstract}

\section{INTRODUCTION}

In the last years, there has been an interest in the study and classification of commutative algebras of Toeplitz operators. This is accompanied by the study of the underlying geometric structures associated to the corresponding symbols. Some previous works along these lines can be found in the references. In this work, we continue the investigation of such topic by introducing quasi-homogeneous quasiradial symbols on a family of weakly pseudo-convex domains $\Omega_{p}^{n}$ (see the definitions below). Such family of domains contains the unit ball $\mathbb{B}^{n}$ as a particular case. Furthermore, our symbols generalize those considered in [12] for the unit ball $\mathbb{B}^{n}$ as well as those considered in $\left[8\right.$ for the complex projective space $\mathbb{P}^{n}(\mathbb{C})$. The sets of symbols introduced in this work are shown to define commutative Banach algebras of Toeplitz operators, which are not $C^{*}$, in the Bergman space of $\Omega_{p}^{n}$. In [9] it was shown the existence of "large" commutative $C^{*}$-algebras of Toeplitz on Reinhardt domains. With this respect, the commutative algebras of Toeplitz operators constructed in this work are the first Banach not $C^{*}$ examples defined on Reinhardt domains that are not bounded symmetric.

As in most of the works that precede this one, it turns out that the symbols that define our commuting Toeplitz operators have a strong geometric background. In fact, we prove that our quasi-homogeneous quasi-radial symbols have an associated group of isometries characterized as the largest connected subgroup leaving invariant the symbols; this is among the toral isometries of $\Omega_{p}^{n}$ as Reinhardt domain (see Theorem 5.1). We also obtain Lagrangian frames for the set of symbols which are defined on the leaves of a foliation of $\Omega_{p}^{n}$ (see Theorem 5.8).

It is worthwhile to mention that this work provides new results for the case of the unit ball $\mathbb{B}^{n}$. More precisely, since our quasi-homogeneous quasi-radial symbols

1991 Mathematics Subject Classification. Primary 47B35; Secondary 32A36, 32M15, 53C12.

Key words and phrases. Toeplitz operator, Bergman space, commutative Banach algebra, Lagrangian manifolds.

The authors were supported by SNI and a Conacyt grant. 
for $\Omega_{p}^{n}$ generalize those found in [12, our Theorems 5.1 and 5.8 hold for the symbols considered in 12 for $\mathbb{B}^{n}$ (see Remark [5.2). Hence, this work establishes the geometric nature and properties for the quasi-homogeneous quasi-radial symbols introduced by N. Vasilevski in [12].

\section{Preliminaries}

Given a multi-index $\alpha=\left(\alpha_{1}, \alpha_{2}, \ldots, \alpha_{n}\right) \in \mathbb{N}^{n}$ we will use the standard notation

$$
\begin{aligned}
|\alpha| & =\alpha_{1}+\alpha_{2}+\cdots+\alpha_{n}, \\
\alpha ! & =\alpha_{1} ! \alpha_{2} ! \cdots \alpha_{n} ! \\
z^{\alpha} & =z_{1}^{\alpha_{1}} z_{2}^{\alpha_{2}} \cdots z_{n}^{\alpha_{n}} .
\end{aligned}
$$

For $p \in \mathbb{Z}_{+}^{n}$ a fixed multi-index, we define the following sets

$$
\begin{aligned}
& \Omega_{p}^{n}(r)=\left\{\left.\left(z_{1}, \ldots, z_{n}\right) \in \mathbb{C}^{n}\left|\sum_{j=1}^{n}\right| z_{j}\right|^{2 p_{j}}<r^{2}\right\} \\
& \mathbb{S}_{p}^{n}(r)=\left\{\left.\left(z_{1}, \ldots, z_{n}\right) \in \mathbb{C}^{n}\left|\sum_{j=1}^{n}\right| z_{j}\right|^{2 p_{j}}=r^{2}\right\}
\end{aligned}
$$

For the case $r=1$, we simply write $\Omega_{p}^{n}$ and $\mathbb{S}_{p}^{n}$, respectively. Note that for $p=$ $(1, \ldots, 1)$, we have that $\Omega_{p}^{n}=\mathbb{B}^{n}$ is the unit ball and $\mathbb{S}_{p}^{n}=\mathbb{S}^{n}$ is the unit sphere in $\mathbb{C}^{n}$, both centered at the origin.

For every $z \in \mathbb{C}^{n}$, we also denote

$$
\begin{aligned}
r & =\|z\|_{p}=\sqrt{\left|z_{1}\right|^{2 p_{1}}+\cdots+\left|z_{n}\right|^{2 p_{n}}} \\
\xi_{j} & =\frac{z_{j}}{\|z\|_{p}^{\frac{1}{p_{j}}}}=\frac{z_{j}}{r^{\frac{1}{p_{j}}}}
\end{aligned}
$$

for all $j=1, \ldots, n$. In particular we have

$$
\sum_{j=1}^{n}\left|\xi_{j}\right|^{2 p_{j}}=1
$$

which implies that $\xi=\left(\xi_{1}, \ldots, \xi_{n}\right) \in \mathbb{S}_{p}^{n}$. Note that these expressions define a set of coordinates $(r, \xi)$ for every $z \in \mathbb{C}^{n}$, these coordinates are called $p$-polar coordinates.

We let $d v$ denote the Lebesgue measure on $\Omega_{p}^{n}$ normalized so that $v\left(\Omega_{p}^{n}\right)=1$. Also we let $\sigma$ denote the hypersurface measure on $\mathbb{S}_{p}^{n}$, also normalized so that $\sigma\left(\mathbb{S}_{p}^{n}\right)=1$.

It is well known that the measures on these sets are related by the following identity, that holds in the case of $\mathbb{B}^{n}$ and $\mathbb{S}^{n}$,

$$
\int_{\mathbb{B}^{n}} f(z) d v(z)=2 n \int_{0}^{1} r^{2 n-1} \int_{\mathbb{S}^{n}} f(r, \xi) d \sigma(\xi) d r
$$

for every non-negative measurable function $f$ on $\mathbb{B}^{n}$. For more details we refer the reader to 13 . The next result is an extension of this equation for arbitrary $p$ as above.

Lemma 2.1. The measures on $v$ and $\sigma$ satisfy

$$
\int_{\Omega_{p}^{n}} f(z) d v(z)=\left(2 \sum_{j=1}^{n} \frac{1}{p_{j}}\right) \int_{0}^{1} r^{2\left(\sum_{j=1}^{n} \frac{1}{p_{j}}\right)-1} \int_{\mathbb{S}_{p}^{n}} f(r, \xi) d \sigma(\xi) d r .
$$


for every non-negative measurable function $f$ on $\Omega_{p}^{n}$.

Proof. The proof follows the same arguments as those found in [13 for $\mathbb{B}^{n}$ and $\mathbb{S}^{n}$, and we present them for the sake of completeness.

Denote by $d V=d x_{1} d y_{1} \cdots d x_{n} d y_{n}$ the $n$-dimensional Lebesgue measure without normalization. Similarly, let $d S$ denote the hypersurface measure of $\mathbb{S}_{p}^{n}$ before normalization. As usual, these coordinates are obtained by the identification $z_{j}=$ $x_{j}+i y_{j}$. Then, the Lebesgue measure of the solid given by by $d S$ in $\mathbb{S}_{p}^{n}$ together with $r>0$ and $r+d r$, is given by

$$
d V=\frac{d S}{S(1)}(V(r+d r)-V(r))
$$

where $V(r)$ in the Lebesgue measure of $\Omega_{p}^{n}(r)$ and $S(r)$ is the is the measure of $\mathbb{S}_{p}^{n}(r)$.

The change of variables $z_{j}=r^{\frac{1}{p_{j}}} \xi_{j}$ yields

$$
V(r)=\int_{\Omega_{p}^{n}(r)} d V(z)=r^{2 \sum_{j=1}^{n} \frac{1}{p_{j}}} V(1)
$$

and so it follows that

$$
d V=\frac{V(1) d S}{S(1)}\left((r+d r)^{2 \sum_{j=1}^{n} \frac{1}{p_{j}}}-r^{2 \sum_{j=1}^{n} \frac{1}{p_{j}}}\right) .
$$

This proves that

$$
d V=\frac{V(1)}{S(1)}\left(2 \sum_{j=1}^{n} \frac{1}{p_{j}}\right) r^{2\left(\sum_{j=1}^{n} \frac{1}{p_{j}}\right)-1} d r d S
$$

which clearly implies

$$
d v=\left(2 \sum_{j=1}^{n} \frac{1}{p_{j}}\right) r^{2\left(\sum_{j=1}^{n} \frac{1}{p_{j}}\right)-1} d r d \sigma
$$

where $d v$ and $d \sigma$ are the normalized measures of $\Omega_{p}^{n}$ and $\mathbb{S}_{p}^{n}$, respectively.

The Hilbert spaces $L^{2}\left(\Omega_{p}^{n}\right)$ and $L^{2}\left(S_{p}^{n}\right)$ are those associated to the usual Lebesgue measure $d V$ on $\Omega_{p}^{n}$ and the hypersurface measure $d S$ on $S_{p}^{n}$. We denote by $\mathcal{A}^{2}\left(\Omega_{p}^{n}\right)$ the closed subspace of $L^{2}\left(\Omega_{p}^{n}\right)$ consisting of those functions which are holomorphic in $\Omega_{p}^{n}$, and we let $P_{p}: L^{2}\left(\Omega_{p}^{n}\right) \rightarrow \mathcal{A}^{2}\left(\Omega_{p}^{n}\right)$ be the orthogonal projection. If $a \in L_{\infty}\left(\Omega_{p}^{n}\right)$ then the Toeplitz operator $T_{a}$ with symbol $a$ is the bounded operator on $\mathcal{A}^{2}\left(\Omega_{p}^{n}\right)$ defined by $T_{a}(f)=P_{p}(a f)$.

We have following identity, and for its proof we refer to [1].

$$
\left\langle z^{\alpha}, z^{\beta}\right\rangle=\delta_{\alpha, \beta} \frac{\pi^{n} \prod_{j=1}^{n} \Gamma\left(\frac{\alpha_{j}+1}{p_{j}}\right)}{\left[\prod_{j=1}^{n} p_{j}\right] \Gamma\left(\sum_{j=1}^{n} \frac{\alpha_{j}+1}{p_{j}}+1\right)} .
$$


In particular, we also have

$$
C_{p}^{n}:=V(1)=\frac{\pi^{n} \prod_{j=1}^{n} \Gamma\left(\frac{1}{p_{j}}\right)}{\left[\prod_{j=1}^{n} p_{j}\right] \Gamma\left(\sum_{j=1}^{n} \frac{1}{p_{j}}+1\right)}
$$

As a consequence of (2.4) we obtain an orthonormal basis for $\mathcal{A}^{2}\left(\Omega_{p}^{n}\right)$ given by

$$
\left(\frac{\left[\prod_{j=1}^{n} p_{j}\right] \Gamma\left(\sum_{j=1}^{n} \frac{\alpha_{j}+1}{p_{j}}+1\right)}{\pi^{n} \prod_{j=1}^{n} \Gamma\left(\frac{\alpha_{j}+1}{p_{j}}\right)}\right)^{\frac{1}{2}} z^{\alpha}
$$

When $p=(1, \ldots, 1)$ and for every $\alpha, \beta \in \mathbb{N}^{n}$ we have

$$
\int_{\mathbb{S}^{n}} \xi^{\alpha} \overline{\xi^{\beta}} d S(\xi)=\delta_{\alpha, \beta} \frac{2 \pi^{n} \alpha !}{(n-1+|\alpha|) !} .
$$

For a proof of this identity we refer to [13]. Such identity has the the following extension for arbitrary $p \in \mathbb{N}^{n}$.

Lemma 2.2. Let $p \in \mathbb{Z}_{+}^{n}$ and $\alpha, \beta \in \mathbb{N}^{n}$. Then

$$
\int_{\mathbb{S}_{p}^{n}} \xi^{\alpha \overline{\xi^{\beta}}} d \sigma(\xi)=\delta_{\alpha, \beta} \frac{\Gamma\left(\sum_{j=1}^{n} \frac{1}{p_{j}}\right) \prod_{j=1}^{n} \Gamma\left(\frac{\alpha_{j}+1}{p_{j}}\right)}{\left[\prod_{j=1}^{n} \Gamma\left(\frac{1}{p_{j}}\right)\right] \Gamma\left(\sum_{j=1}^{n} \frac{\alpha_{j}+1}{p_{j}}\right)},
$$

or equivalently

$$
\int_{\mathbb{S}_{p}^{n}} \xi^{\alpha} \overline{\xi^{\beta}} d S(\xi)=\delta_{\alpha, \beta} \frac{2 \pi^{n} \prod_{j=1}^{n} \Gamma\left(\frac{\alpha_{j}+1}{p_{j}}\right)}{\left[\prod_{j=1}^{n} p_{j}\right] \Gamma\left(\sum_{j=1}^{n} \frac{\alpha_{j}+1}{p_{j}}\right)}
$$

Proof. As a consequence of the rotation invariance of the measure on $\mathbb{S}_{p}^{n}$ it follows that

$$
\int_{\mathbb{S}_{p}^{n}} \xi^{\alpha} \overline{\xi^{\beta}} d \sigma(\xi)=0
$$

for every $\alpha \neq \beta$. Following [13], we evaluate the integral

$$
I=\int_{\mathbb{C}^{n}}\left|z^{\alpha}\right|^{2} e^{-\left(\left|z_{1}\right|^{2 p_{1}}+\cdots+\left|z_{n}\right|^{2 p_{n}}\right)} d V
$$

by two diferents methods. First, Fubini's theorem gives

$$
I=\int_{\mathbb{C}^{n}}\left|z^{\alpha}\right|^{2} e^{-\left(\left|z_{1}\right|^{2 p_{1}}+\cdots+\left|z_{n}\right|^{2 p_{n}}\right)} d V
$$




$$
\begin{aligned}
& =\prod_{j=1}^{n} \int_{\mathbb{R}^{n}}\left(x^{2}+y^{2}\right)^{\alpha_{j}} e^{-\left(x^{2}+y^{2}\right)^{p_{j}}} d x d y \\
& =\pi^{n} \prod_{j=1}^{n} \int_{0}^{\infty} r^{\alpha_{j}} e^{-r^{p_{j}}} d r .
\end{aligned}
$$

Using the change of variables $r=s^{\frac{1}{p_{j}}}$, for which $d r=\frac{1}{p_{j}} s^{\frac{1}{p_{j}}-1} d s$, we obtain

$$
\begin{aligned}
I & =\pi^{n} \prod_{j=1}^{n} \frac{1}{p_{j}} \int_{0}^{\infty} s^{\frac{\alpha_{j}+1}{p_{j}}-1} e^{-s} d s \\
& =\pi^{n} \prod_{j=1}^{n} \frac{1}{p_{j}} \Gamma\left(\frac{\alpha_{j}+1}{p_{j}}\right)
\end{aligned}
$$

On the other hand, we integrate in $p$-polar cordinates

$$
I=2 \sum_{j=1}^{n} \frac{1}{p_{j}} C_{p}^{n} \int_{0}^{\infty} r^{2\left(\sum_{j=1}^{n} \frac{\alpha_{j}+1}{p_{j}}\right)-1} e^{-r^{2}} d r \int_{\mathbb{S}_{p}^{n}}\left|\xi^{\alpha}\right|^{2} d \sigma(\xi)
$$

where $\xi_{j}=\frac{z_{j}}{r^{\frac{1}{p_{j}}}}$, for all $j=1, \ldots, n$. If we take $r=\sqrt{s}$, then $d r=\frac{1}{2} s^{-\frac{1}{2}} d s$, and so

$$
I=2\left(\sum_{j=1}^{n} \frac{1}{p_{j}}\right) C_{p}^{n} \int_{0}^{\infty} s^{\left(\sum_{j=1}^{n} \frac{\alpha_{j}+1}{p_{j}}\right)-1} \frac{d s}{2} \int_{\mathbb{S}_{p}^{n}}\left|\xi^{\alpha}\right|^{2} d \sigma(\xi)
$$

which is equivalent to

$$
I=\sum_{j=1}^{n} \frac{1}{p_{j}} C_{p}^{n} \Gamma\left(\sum_{j=1}^{n} \frac{\alpha_{j}+1}{p_{j}}\right) \int_{\mathbb{S}_{p}^{n}}\left|\xi^{\alpha}\right|^{2} d \sigma(\xi) .
$$

From (2.9) and (2.10) we obtain the identity

$$
\pi^{n} \prod_{j=1}^{n} \frac{1}{p_{j}} \Gamma\left(\frac{\alpha_{k}+1}{p_{j}}\right)=\sum_{j=1}^{n} \frac{1}{p_{j}} C_{p}^{n} \Gamma\left(\sum_{j=1}^{n} \frac{\alpha_{k}+1}{p_{j}}\right) \int_{\mathbb{S}_{p}^{n}}\left|\xi^{\alpha}\right|^{2} d \sigma(\xi) .
$$

Hence, we conclude that

$$
\begin{aligned}
\int_{\mathbb{S}_{p}^{n}}\left|\xi^{\alpha}\right|^{2} d \sigma(\xi) & =\frac{\pi^{n} \prod_{j=1}^{n}\left[\frac{1}{p_{j}} \Gamma\left(\frac{\alpha_{j}+1}{p_{j}}\right)\right]}{C_{p}^{n}\left(\sum_{j=1}^{n} \frac{1}{p_{j}}\right) \Gamma\left(\sum_{j=1}^{n} \frac{\alpha_{j}+1}{p_{j}}\right)} \\
& =\frac{\left(\prod_{j=1}^{n} p_{j}\right) \Gamma\left(\sum_{j=1}^{n} \frac{1}{p_{j}}+1\right) \prod_{j=1}^{n}\left[\frac{1}{p_{j}} \Gamma\left(\frac{\alpha_{j}+1}{p_{j}}\right)\right]}{\left[\prod_{j=1}^{n} \Gamma\left(\frac{1}{p_{j}}\right)\right]\left(\sum_{j=1}^{n} \frac{1}{p_{j}}\right) \Gamma\left(\sum_{j=1}^{n} \frac{\alpha_{j}+1}{p_{j}}\right)}
\end{aligned}
$$




$$
=\frac{\Gamma\left(\sum_{j=1}^{n} \frac{1}{p_{j}}\right) \prod_{j=1}^{n} \Gamma\left(\frac{\alpha_{j}+1}{p_{j}}\right)}{\left[\prod_{j=1}^{n} \Gamma\left(\frac{1}{p_{j}}\right)\right] \Gamma\left(\sum_{j=1}^{n} \frac{\alpha_{j}+1}{p_{j}}\right)},
$$

which proves (2.7).

We now recall that the volume of $\Omega_{p}^{n}(r)$ is given by (2.3) and that

$$
V(r)=\int_{0}^{r} S(x) d x
$$

where $S(x)$ is the volume of $\mathbb{S}_{p}^{n}(x)$. It follows that

$$
S(r)=V^{\prime}(r)=\left(2 \sum_{j=1}^{n} \frac{1}{p_{j}}\right) r^{2 \sum_{j=1}^{n} \frac{1}{p_{j}}-1} V(1)
$$

In particular the volume of $\mathbb{S}_{p}^{n}$ is given by

$$
S(1)=\left(2 \sum_{j=1}^{n} \frac{1}{p_{j}}\right) V(1)=\frac{2 \pi^{n} \prod_{j=1}^{n} \Gamma\left(\frac{1}{p_{j}}\right)}{\left[\prod_{j=1}^{n} p_{j}\right] \Gamma\left(\sum_{j=1}^{n} \frac{1}{p_{j}}\right)}
$$

Using this identity and (2.7) we obtain (2.8).

\section{TOEPLITZ OPERATORS WITH QUASI-HOMOGENEUOS SYMBOLS}

Let $k=\left(k_{1}, \ldots, k_{s}\right) \in \mathbb{Z}_{+}^{s}$ be a partition of $n$; in other words we have $|k|=$ $k_{1}+\ldots+k_{s}=n$. Given such $k$ we define

$$
\widehat{k}_{j}= \begin{cases}0 & \text { if } j=0, \\ \widehat{k}_{j-1}+k_{j} & \text { if } j=1, \ldots, s\end{cases}
$$

Given $z \in \mathbb{C}^{n}$ and $k \in \mathbb{Z}_{+}^{s}$ a partition of $n$ we decompose $z$ into $s$ pieces, each one of which has $k_{j}$ components for $j=1, \ldots, s$. This is achieved by denoting

$$
z_{(j)}=\left(z_{\widehat{k}_{j-1}}, \ldots, z_{\widehat{k}_{j}}\right)
$$

for $j=1, \ldots, s$. In particular, $z_{(j)} \in \mathbb{C}^{k_{j}}$ for every $j=1, \ldots s$ and $z=\left(z_{(1)}, \ldots z_{(s)}\right)$ for every $z \in \mathbb{C}^{n}$. We also note that the components of $z_{(j)} \in \mathbb{C}^{k_{j}}$ are given by $\left(z_{(j)}\right)_{1}, \ldots,\left(z_{(j)}\right)_{k_{j}}$.

Now let us choose $p \in \mathbb{Z}_{+}^{n}$ as before. Then, we denote

$$
r_{j}=\left\|z_{(j)}\right\|_{p}=\sqrt{\sum_{t=\widehat{k}_{j-1}+1}^{\widehat{k}_{j}}\left|z_{t}\right|^{2 p_{t}}}
$$


for every $j=1, \ldots, s$. Furthermore, for every $z \in \mathbb{C}^{n}$ we denote

$$
\xi_{(j)}=\left(\frac{\left(z_{(j)}\right)_{1}}{r_{j}^{\overline{\left.p_{(j)}\right)} 1}}, \ldots, \frac{\left(z_{(j)}\right) k_{j}}{r_{j}^{\left(p_{(j)}\right)_{k_{j}}}}\right),
$$

for every $j=1, \ldots, s$. In words, the element $\xi_{(j)}$ is obtained from $z_{(j)}$ by dividing each component of the latter by $r_{j}=\left\|z_{(j)}\right\|_{p}$ powered to the exponents given by the reciprocals of the corresponding components of $p_{(j)}$. Note that $\xi_{(j)} \in \mathbb{S}_{p_{(j)}}^{k_{j}}$ for every $j$.

Definition 3.1. Let $k=\left(k_{1}, \ldots, k_{s}\right) \in \mathbb{Z}_{+}^{s}$ be a partition of $n$ and let $\nu, \mu \in \mathbb{N}^{n}$ be such that

$$
\nu \cdot \mu=\nu_{1} \mu_{0}+\cdots+\nu_{n} \mu_{n}=0 .
$$

With the above notation, the $k$-quasi-homogeneous symbol associated to $\nu, \mu$ is the function $\varphi: \Omega_{p}^{n} \rightarrow \mathbb{C}$ given by

$$
\varphi(z)=\xi^{\nu} \bar{\xi}^{\mu}=\prod_{j=0}^{s}\left(\xi_{(j)}\right)^{\nu_{(j)}}\left(\xi_{(j)}\right)^{\mu_{(j)}} .
$$

We will denote by $\mathcal{H}_{k}\left(\Omega_{p}^{n}\right)$ the set of $k$-quasi-homogeneous symbols on $\Omega_{p}^{n}$.

It is a simple matter to see that for $p=(1, \ldots, 1)$ a $k$-quasi-homogeneous symbol on $\Omega_{p}^{n}=\mathbb{B}^{n}$ is $k$-quasi-homogeneous in the sense of $[12$.

Definition 3.2. Let $k=\left(k_{1}, \ldots, k_{s}\right) \in \mathbb{Z}_{+}^{s}$ be a partition of $n$. With the above notation, a $k$-quasi-radial symbol is a function function $a: \Omega_{p}^{n} \rightarrow \mathbb{C}$ that can be written as $a(z)=\widetilde{a}\left(r_{1}, \ldots, r_{s}\right)$ where $r_{j}$ is given by 3.1 . We will denote by $\mathcal{R}_{k}\left(\Omega_{p}^{n}\right)$ the set of $k$-quasi-radial symbols on $\Omega_{p}^{n}$.

As before, it is easy to see that for $p=(1, \ldots, 1)$ a $k$-quasi-radial symbol on $\Omega_{p}^{n}=\mathbb{B}^{n}$ is $k$-quasi-radial in the sense of 12 .

The two definitions above yield together the notion of quasi-homogeneous quasiradial symbol.

Definition 3.3. Let $k=\left(k_{1}, \ldots, k_{s}\right) \in \mathbb{Z}_{+}^{s}$ be a partition of $n$. Then, a $k$-quasihomogeneous quasi-radial symbol is a function $\Omega_{p}^{n} \rightarrow \mathbb{C}$ of the form $a \varphi$, where $a \in \mathcal{R}_{k}\left(\Omega_{p}^{n}\right)$ and $\varphi \in \mathcal{H}_{k}\left(\Omega_{p}^{n}\right)$; in this case, we will refer to $a$ and $\varphi$ as the quasiradial and quasi-homogeneous parts of the symbol, respectively. We will denote by $\mathcal{H} \mathcal{R}_{k}\left(\Omega_{p}^{n}\right)$ the set of $k$-quasi-homogeneous quasi-radial symbols.

We observe that the domain $\Omega_{p}^{n}$ is a Reinhardt domain and so the results from [9] can be applied in this case. In particular, 9] implies that the set symbols $\mathcal{R}_{(1, \ldots, 1)}$ defines a family of commutative Toeplitz operators on $\mathcal{A}^{2}\left(\Omega_{p}^{n}\right)$. Furthermore, every Toeplitz operator $T_{a}$ with symbol $a \in \mathcal{R}_{k}\left(\Omega_{p}^{n}\right)$ is diagonal with respect to the standard monomial basis. Since $\mathcal{R}_{k}\left(\Omega_{p}^{n}\right)$ is a subset of $\mathcal{R}_{(1, \ldots, 1)}\left(\Omega_{p}^{n}\right)$, the same properties hold for $\mathcal{R}_{k}\left(\Omega_{p}^{n}\right)$. The following result provides an explicit presentation of such Toeplitz operators as diagonalizable operators.

Lemma 3.4. Let $k \in \mathbb{Z}_{+}^{s}$ be a partition of $n$. Then, for any $k$-quasi-radial bounded measurable symbol $a \in \mathcal{R}_{k}\left(\Omega_{p}^{n}\right)$, we have

$$
T_{a} z^{\alpha}=\gamma_{a, k}(\alpha) z^{\alpha}
$$


for every $\alpha \in \mathbb{N}^{n}$, where

$$
\begin{aligned}
\gamma_{a, k}(\alpha) & =\frac{4^{s} \Gamma\left(\sum_{j=1}^{n} \frac{\alpha_{j}+1}{p_{j}}+1\right) \prod_{j=1}^{s}\left(\sum_{t=\widehat{k}_{j-1}+1}^{\widehat{k}_{j}} \frac{1}{p_{t}}\right)}{\prod_{j=1}^{s} \Gamma\left(\sum_{t=\widehat{k}_{j-1}+1}^{\widehat{k}_{j}} \frac{\alpha_{t}+1}{p_{t}}\right)} \\
& \times \int_{\Delta_{s}^{n}} a\left(r_{1}, \ldots, r_{s}\right) \prod_{j=1}^{s} r_{j}^{\left(2 \sum_{t=\widehat{k}_{j-1}+1}^{\widehat{k}_{j}}\left(\frac{\alpha_{t}+1}{p_{t}}\right)-1\right)} d r_{j}
\end{aligned}
$$

and $\Delta_{s}^{n}=\left\{\left(r_{1}, \ldots, r_{s}\right) \in \mathbb{R}_{+}: r_{1}^{2}+\cdots+r_{s}^{2}<1\right\}$.

Proof. Let $\alpha \in \mathbb{N}^{n}$. Then, we have

$$
\left\langle T_{a} z^{\alpha}, z^{\alpha}\right\rangle=\left\langle a\left(r_{1}, \ldots, r_{s}\right) z^{\alpha}, z^{\alpha}\right\rangle=\int_{\Omega_{p}^{n}} a\left(r_{1}, \ldots, r_{s}\right)\left|z^{\alpha}\right|^{2} d V
$$

Consider the change of variables $\left(z_{(j)}\right)_{t}=r_{j}^{\frac{1}{p_{(j), t}}}\left(\xi_{(j)}\right)_{t}$, for every $t=1, \ldots, k_{j}$ and $j=1, \ldots, s$. Then, we obtain

$$
\begin{aligned}
\left\langle a\left(r_{1}, \ldots, r_{s}\right) z^{\alpha}, z^{\alpha}\right\rangle & =\int_{\Delta_{s}^{n}} a\left(r_{1}, \ldots, r_{s}\right) \prod_{j=1}^{s} r_{j}^{\left[2 \sum_{t=\widehat{k}_{j-1}+1}^{\widehat{k}_{j}}\left(\frac{\alpha_{t}+1}{p_{t}}\right)-1\right]} d r_{j} \\
& \times \prod_{j=1}^{s} 2\left(\sum_{t=\widehat{k}_{j-1}}^{\widehat{k}_{j}} \frac{1}{p_{t}}\right) \int_{S_{(j)}^{k_{j}}}\left|\xi_{(j)}\right|^{\alpha_{(j)}} d S_{j} \\
& =\int_{\Delta_{s}^{n}} a\left(r_{1}, \ldots, r_{s}\right) \prod_{j=1}^{s} r_{j}^{\left[2 \sum_{t=\widehat{k}_{j-1}+1}^{\widehat{k}_{j}}\left(\frac{\alpha_{t}+1}{p_{t}}\right)-1\right]} d r_{j} \\
& \times \frac{4^{s} \pi^{n} \prod_{j=1}^{n} \Gamma\left(\frac{\alpha_{j}+1}{p_{j}}\right) \prod_{j=1}^{s}\left(\sum_{t=\widehat{k}_{j-1}+1}^{\widehat{k}_{j}} \frac{1}{p_{t}}\right)}{\prod_{j=1}^{s} \Gamma\left(\sum_{t=\widehat{k}_{j-1}+1}^{\widehat{k}_{j}} \frac{\alpha_{t}+1}{p_{t}}\right) \prod_{j=1}^{n} p_{j}},
\end{aligned}
$$

and the result follows from (2.4).

We now determine the action on monomials of the Toeplitz operators with symbols in $\mathcal{H} \mathcal{R}_{k}\left(\Omega_{p}^{n}\right)$.

Lemma 3.5. Let $k \in \mathbb{Z}_{+}^{s}$ be a partition of $n$ and $\nu, \mu \in \mathbb{N}^{n}$. If a $\xi^{\nu} \bar{\xi}^{\mu}=a\left(r_{1}, \ldots, r_{l}\right) \xi^{\nu} \bar{\xi}^{\mu}$ is an element of $\mathcal{H} \mathcal{R}_{k}\left(\Omega_{p}^{n}\right)$, then the Toeplitz operator $T_{a \xi^{\nu} \bar{\xi}^{\mu}}$ acts on monomials $z^{\alpha}$ with $\alpha \in \mathbb{N}^{n}$ as follows

$$
T_{a \xi^{\nu} \bar{\xi}^{\mu}} z^{\alpha}= \begin{cases}\tilde{\gamma}_{a, k, \nu, \mu}(\alpha) z^{\alpha+\nu-\mu} & \text { for } \alpha+\nu-\mu \in \mathbb{Z}_{+}^{n} \\ 0 & \text { for } \alpha+\nu-\mu \notin \mathbb{Z}_{+}^{n}\end{cases}
$$

where 


$$
\begin{gathered}
\tilde{\gamma}_{a, k, \nu, \mu}(\alpha)=\int_{\Delta_{s}^{n}} a\left(r_{1}, \ldots, r_{s}\right) \prod_{j=1}^{s} r_{j}^{\left(\sum_{t=\widehat{k}_{j-1}+1}^{\widehat{k}_{j}} \frac{2 \alpha_{t}+\nu_{t}-\mu_{t}+2}{p_{t}}-1\right)} d r_{j} \\
\times \frac{4^{s} \prod_{j=1}^{n} \Gamma\left(\frac{\alpha_{j}+\nu_{j}+1}{p_{j}}\right) \prod_{j=1}^{s}\left(\sum_{t=\widehat{k}_{j-1}+1}^{\widehat{k}_{j}} \frac{1}{p_{t}}\right) \Gamma\left(\sum_{j=1}^{n} \frac{\alpha_{j}+\nu_{j}-\mu_{j}+1}{p_{j}}+1\right)}{\prod_{j=1}^{n} \Gamma\left(\frac{\alpha_{j}+\nu_{j}-\mu_{k}+1}{p_{j}}\right) \prod_{j=1}^{s} \Gamma\left(\sum_{t=\widehat{k}_{j-1}+1}^{\widehat{k}_{j}} \frac{\alpha_{t}+\nu_{t}+1}{p_{t}}\right)}
\end{gathered}
$$

Proof. Let $\alpha, \beta \in \mathbb{N}^{n}$. Then, we have

$$
\begin{aligned}
\left\langle T_{a \xi^{\nu} \bar{\xi}^{\mu}} z^{\alpha}, z^{\beta}\right\rangle & =\left\langle a \xi^{\nu} \bar{\xi}^{\mu} z^{\alpha}, z^{\beta}\right\rangle \\
& =\int_{\Omega_{p}^{n}} a\left(r_{1}, \ldots, r_{s}\right) \xi^{\nu} \bar{\xi}^{\mu} z^{\alpha} \bar{z}^{\beta} d V
\end{aligned}
$$

Consider the change of variables $\left(z_{(j)}\right)_{t}=r_{j}^{\frac{1}{p(j), t}}\left(\xi_{(j)}\right)_{t}$, for $t=1, \ldots, k_{j}$ and $j=1, \ldots, s$. Then, we obtain

$$
\begin{aligned}
\left\langle T_{\left.a \xi^{\nu} \bar{\xi}^{\mu} z^{\alpha}, z^{\beta}\right\rangle=} \int_{\Delta_{s}^{n}} a\left(r_{1}, \ldots, r_{s}\right) \prod_{j=1}^{s} 2\left(\sum_{t=\widehat{k}_{j-1}+1}^{\widehat{k}_{j}} \frac{1}{p_{t}}\right)\right. \\
\quad \times r_{j}^{\left(\sum_{t=\widehat{k}_{j-1}+1}^{\widehat{k}_{j}} \frac{\alpha_{t}+\beta_{t}+2}{p_{t}}-1\right)} d r_{j} \\
\times \prod_{j=1}^{s} \int_{S_{p_{(j)}}^{k_{j}}} \xi^{\nu_{(j)}+\alpha_{(j)} \bar{\xi}^{\mu_{(j)}+\beta_{(j)}} d S_{j}} \\
=\delta_{\alpha+\nu, \beta+\mu} \int_{\Delta_{s}^{n}} a\left(r_{1}, \ldots, r_{s}\right) \prod_{j=1}^{s} r_{j}^{\left[\sum_{t=\widehat{k}_{j-1}+1}^{\widehat{k}_{j}} \frac{\alpha_{t}+\beta_{t}+2}{p_{t}}-1\right]} d r_{j} \\
\times \\
\quad \frac{4^{s} \pi^{n} \prod_{t=1}^{n} \Gamma\left(\frac{\alpha_{t}+\nu_{t}+1}{p_{t}}\right) \prod_{j=1}^{s}\left(\sum_{t=\widehat{k}_{j-1}+1}^{\widehat{k}_{j}} \frac{1}{p_{t}}\right)}{\prod_{j=1}^{n} p_{j} \prod_{j=1}^{s} \Gamma\left(\sum_{t=\widehat{k}_{j-1}+1}^{\widehat{k}_{j}} \frac{\alpha_{t}+\nu_{t}+1}{p_{t}}\right)}
\end{aligned}
$$

Observe that this expression is non zero if and only if $\beta=\alpha+\nu-\mu$, which a priori belongs to $\mathbb{N}^{n}$. We conclude the result from the orthonormality of the basis defined in (2.6).

\section{Commutativity Results For QUASI-HOMOGEneuos SYmbols}

We obtain commutativity results for Toeplitz operators on the domain $\Omega_{p}^{n}$ that extend those found in [12].

Theorem 4.1. Let $k=\left(k_{1}, \ldots, k_{s}\right) \in \mathbb{Z}_{+}^{s}$ be a partition of $n$ and $\nu, \mu \in \mathbb{N}^{n}$ a pair of orthogonal multi-indices. Let $a_{1}, a_{2} \in \mathcal{R}_{k}$ be non identically zero and 
$\xi^{\nu} \bar{\xi}^{\mu} \in \mathcal{H}_{k}$. Then, the Toeplitz operators $T_{a_{1}}$ and $T_{a_{2} \xi^{\nu} \bar{\xi}^{\mu}}$ commute on the Bergman space $\mathcal{A}^{2}\left(\Omega_{p}^{n}\right)$ if and only if

$$
\sum_{t=\widehat{k}_{j-1}+1}^{\widehat{k}_{j}} \frac{\nu_{t}-\mu_{t}}{p_{t}}=\sum_{t=1}^{k_{j}} \frac{\left(\nu_{(j)}\right)_{t}-\left(\mu_{(j)}\right)_{t}}{\left(p_{(j)}\right)_{t}}=0
$$

for each $j=1, \ldots, s$.

Proof. Let $\alpha \in \mathbb{Z}_{+}^{n}$ be given. First note that if $\alpha+\nu-\mu \notin \mathbb{Z}_{+}^{n}$, then the Lemmas 3.4 and 3.5 imply that both $T_{a_{1}} T_{a_{2} \xi^{\nu}} \bar{\xi}^{\mu} z^{\alpha}$ and $T_{a_{2} \xi^{\nu}} \bar{\xi}^{\mu} T_{a_{1}} z^{\alpha}$ vanish. Hence, we can assume that $\alpha+\nu-\mu \in \mathbb{Z}_{+}^{n}$.

Applying again Lemmas 3.4 and 3.5 we obtain

$$
\begin{aligned}
& T_{a_{1}} T_{a_{2} \xi^{\nu} \bar{\xi}^{\mu}} z^{\alpha}= \\
& \frac{4^{s} \prod_{t=1}^{n} \Gamma\left(\frac{\alpha_{t}+\nu_{t}+1}{p_{t}}\right) \prod_{j=1}^{s}\left(\sum_{t=\widehat{k}_{j-1}+1}^{\widehat{k}_{j}} \frac{1}{p_{t}}\right) \Gamma\left(\sum_{j=1}^{n} \frac{\alpha_{j}+\nu_{j}-\mu_{j}+1}{p_{j}}+1\right)}{\prod_{j=1}^{n} \Gamma\left(\frac{\alpha_{j}+\nu_{j}-\mu_{j}+1}{p_{j}}\right) \prod_{j=1}^{s} \Gamma\left(\sum_{t=\widehat{k}_{j-1}+1}^{\widehat{k}_{j}} \frac{\alpha_{t}+\nu_{t}+1}{p_{t}}\right)} \\
& \times \int_{\Delta_{s}^{n}} a_{2}\left(r_{1}, \ldots, r_{n}\right) \prod_{j=1}^{s} r_{j}^{\left(\sum_{t=\widehat{k}_{j-1}+1}^{\widehat{k}_{j}} \frac{2 \alpha_{t}+\nu_{t}-\mu_{t}+2}{p_{t}}-1\right)} d r_{j} \\
& \times \frac{4^{s} \Gamma\left(\sum_{j=1}^{n} \frac{\alpha_{j}+\nu_{j}-\mu_{j}+1}{p_{j}}+1\right) \prod_{j=1}^{s}\left(\sum_{t=\widehat{k}_{j-1}}^{\widehat{k}_{j}} \frac{1}{p_{t}}\right)}{\prod_{j=1}^{s} \Gamma\left(\sum_{t=\widehat{k}_{j-1}+1}^{\widehat{k}_{j}} \frac{\alpha_{t}+\nu_{t}-\mu_{t}+1}{p_{t}}\right)} \\
& \times \int_{\Delta_{s}^{n}} a_{1}\left(r_{1}, \ldots, r_{m}\right) \prod_{j=1}^{m} r_{j}^{\left(2 \sum_{t=\widehat{k}_{j-1}+1}^{\widehat{k}_{j}}\left(\frac{\alpha_{t}+\nu_{t}-\mu_{t}+1}{p_{t}}\right)-1\right)} d r_{j} \\
& \times z^{\alpha+\nu-\mu} \text {. }
\end{aligned}
$$

And similarly, we have

$$
\begin{aligned}
& T_{a_{2} \xi^{\nu} \bar{\xi}^{\mu}} T_{a_{1}} z^{\alpha}= \\
& \frac{4^{s} \Gamma\left(\sum_{j=1}^{n} \frac{\alpha_{j}+1}{p_{j}}+1\right) \prod_{j=1}^{s}\left(\sum_{t=\widehat{k}_{j-1}}^{\widehat{k}_{j}} \frac{1}{p_{t}}\right)}{\prod_{j=1}^{s} \Gamma\left(\sum_{t=\widehat{k}_{j-1}+1}^{\widehat{k}_{j}} \frac{\alpha_{t}+1}{p_{t}}\right)} \\
& \times \int_{\Delta_{s}^{n}} a_{1}\left(r_{1}, \ldots, r_{m}\right) \prod_{j=1}^{s} r_{j}^{\left(2 \sum_{t=\widehat{k}_{j-1}+1}^{\widehat{k}_{j}}\left(\frac{\alpha_{t}+1}{p_{t}}\right)-1\right)} d r_{j}
\end{aligned}
$$




$$
\begin{aligned}
& \times \frac{4^{s} \prod_{t=1}^{n} \Gamma\left(\frac{\alpha_{t}+\nu_{t}+1}{p_{t}}\right) \prod_{j=1}^{s}\left(\sum_{t=\widehat{k}_{j-1}+1}^{\widehat{k}_{j}} \frac{1}{p_{t}}\right)}{\prod_{j=1}^{n} \Gamma\left(\frac{\alpha_{j}+\nu_{j}-\mu_{j}+1}{p_{j}}\right)} \\
& \times \frac{\Gamma\left(\sum_{j=1}^{n} \frac{\alpha_{j}+\nu_{j}-\mu_{j}+1}{p_{j}}+1\right)}{\prod_{j=1}^{s} \Gamma\left(\sum_{t=\widehat{k}_{j-1}+1} \frac{\alpha_{t}+\nu_{t}+1}{p_{t}}\right)} \\
& \times \int_{\Delta_{s}^{n}} a_{2}\left(r_{1}, \ldots, r_{n}\right) \prod_{j=1}^{s} r_{j}^{\left(\sum_{t=\widehat{k}_{j-1}+1}^{\widehat{k}_{j}} \frac{2 \alpha_{t}+\nu_{t}-\mu_{t}+2}{p_{t}}-1\right)} d r_{j} \\
& \times z^{\alpha+\nu-\mu}
\end{aligned}
$$

This implies that $T_{a_{1}} T_{a_{2} \xi^{\nu} \bar{\xi}^{\mu}} z^{\alpha}=T_{a_{2} \xi^{\nu} \bar{\xi}^{\mu}} T_{a_{1}} z^{\alpha}$ for all $\alpha$ if and only if

$$
\sum_{t=\widehat{k}_{j-1}+1}^{\widehat{k}_{j}} \frac{\nu_{t}-\mu_{t}}{p_{t}}=0
$$

where $j=1, \ldots, s$.

If we assume that (4.1) holds for all $j=1, \ldots, s$, then equations (3.2) and (3.3) imply the following identity

$$
\begin{aligned}
& \tilde{\gamma}_{a, k, \nu, \mu}(\alpha)= \\
& \int_{\Delta_{m}^{n}} a\left(r_{1}, \ldots, r_{n}\right) \prod_{j=1}^{s} r_{j}^{\left(\sum_{t=\widehat{k}_{j-1}+1}^{\widehat{k}_{j}} \frac{2 \alpha_{t}+2}{p_{t}}-1\right)} d r_{j} \\
& \times \frac{4^{s} \prod_{t=1}^{n} \Gamma\left(\frac{\alpha_{t}+\nu_{t}+1}{p_{t}}\right) \prod_{j=1}^{s}\left(\sum_{t=\widehat{k}_{j-1}+1}^{\widehat{k}_{j}} \frac{1}{p_{t}}\right) \Gamma\left(\sum_{j=1}^{n} \frac{\alpha_{j}+1}{p_{j}}+1\right)}{\prod_{j=1}^{n} \Gamma\left(\frac{\alpha_{j}+\nu_{j}-\mu_{j}+1}{p_{j}}\right) \prod_{j=1}^{s} \Gamma\left(\sum_{t=\widehat{k}_{j-1}+1}^{\widehat{k}_{j}} \frac{\alpha_{t}+\nu_{t}+1}{p_{t}}\right)} \\
& =\frac{\prod_{t=1}^{n} \Gamma\left(\frac{\alpha_{t}+\nu_{t}+1}{p_{t}}\right) \prod_{j=1}^{s} \Gamma\left(\sum_{t=\widehat{k}_{j-1}+1}^{\widehat{k}_{j}} \frac{\alpha_{t}+1}{p_{t}}\right)}{\prod_{j=1}^{n} \Gamma\left(\frac{\alpha_{j}+\nu_{j}-\mu_{j}+1}{p_{j}}\right) \prod_{j=1}^{s} \Gamma\left(\sum_{t=\widehat{k}_{j-1}+1}^{\hat{k}_{j}} \frac{\alpha_{t}+\nu_{t}+1}{p_{t}}\right)} \gamma_{a, k}(\alpha)
\end{aligned}
$$

Theorem 4.2. Let $k=\left(k_{1}, \ldots, k_{s}\right) \in \mathbb{Z}_{+}^{s}$ be a partition of $n$ and let $\nu, \mu, \sigma, \eta \in \mathbb{N}^{n}$ be multi-indices that satisfy the following properties 
- $\nu \perp \mu$ and $\sigma \perp \eta$,

- $\sum_{t=\widehat{k}_{j-1}+1}^{\widehat{k}_{j}} \frac{\nu_{t}-\mu_{t}}{p_{t}}=0$ and $\sum_{t=\widehat{k}_{j-1}+1}^{\widehat{k}_{j}} \frac{\sigma_{t}-\eta_{t}}{p_{t}}=0$ for all $j=0, \ldots, s$.

Let $a \xi^{\nu} \bar{\xi}^{\mu}, b \xi^{\sigma} \bar{\xi}^{\eta} \in \mathcal{H} \mathcal{R}_{k}$ be corresponding $k$-quasi-homogeneous quasi-radial symbols on $\Omega_{p}^{n}$, where $a, b \in \mathcal{R}_{k}$ are measurable and bounded symbols. Then, the Toeplitz operators $T_{a \xi^{\nu} \bar{\xi}^{\mu}}$ and $T_{b \xi^{\sigma} \bar{\xi}^{\eta}}$ commute on the Bergman space $\mathcal{A}^{2}\left(\Omega_{p}^{n}\right)$ if and only if for each $s=1, \ldots, n$ one of the following conditions holds

(1) $\nu_{s}=\mu_{s}=0$

(2) $\sigma_{s}=\eta_{s}=0$

(3) $\nu_{s}=\sigma_{s}=0$

(4) $\mu_{s}=\eta_{s}=0$

Proof. First, we observe that $T_{b \xi^{\sigma} \bar{\xi}^{\eta}} T_{a \xi^{\nu}} \bar{\xi}^{\mu} z^{\alpha}$ and $T_{a \xi^{\nu}} \bar{\xi}^{\mu} T_{b \xi^{\sigma}} \bar{\xi}^{\eta} z^{\alpha}$ are always simultaneously zero or non zero. Hence, we compute such expressions for $\alpha \in N^{n}$ assuming that both are non zero. By (4.2), we have the following expression

$$
\begin{aligned}
& T_{b \xi^{\sigma} \bar{\xi}^{\eta}} T_{a \xi^{\nu} \bar{\xi}^{\mu}} z^{\alpha}= \\
& \int_{\Delta_{m}^{n}} a\left(r_{1}, \ldots, r_{n}\right) \prod_{j=1}^{s} r_{j}^{\left(\sum_{t=\widehat{k}_{j-1}+1}^{\widehat{k}_{j}} \frac{2 \alpha_{t}+2}{p_{t}}-1\right)} d r_{j} \\
& 4^{s} \prod_{t=1}^{n} \Gamma\left(\frac{\alpha_{t}+\nu_{t}+1}{p_{t}}\right) \prod_{j=1}^{s}\left(\sum_{t=\widehat{k}_{j-1}+1}^{\widehat{k}_{j}} \frac{1}{p_{t}}\right) \Gamma\left(\sum_{j=1}^{n} \frac{\alpha_{j}+1}{p_{j}}+1\right) \\
& \prod_{j=1}^{n} \Gamma\left(\frac{\alpha_{j}+\nu_{j}-\mu_{j}+1}{p_{j}}\right) \prod_{j=1}^{s} \Gamma\left(\sum_{t=\widehat{k}_{j-1}+1}^{\widehat{k}_{j}} \frac{\alpha_{t}+\nu_{t}+1}{p_{t}}\right) \\
& \times \int_{\Delta_{m}^{n}} b\left(r_{1}, \ldots, r_{n}\right) \prod_{j=1}^{s} r_{j}^{\left(\sum_{t=\widehat{k}_{j-1}+1}^{\widehat{k}_{j}} \frac{2 \alpha_{t}+2}{p_{t}}-1\right)} d r_{j} \\
& \times \frac{4^{s} \prod_{t=1}^{n} \Gamma\left(\frac{\alpha_{t}+\nu_{t}-\mu_{t}+\sigma_{t}+1}{p_{t}}\right) \prod_{j=1}^{s}\left(\sum_{t=\widehat{k}_{j-1}+1}^{\widehat{k}_{j}} \frac{1}{p_{t}}\right)}{\prod_{j=1}^{n} \Gamma\left(\frac{\alpha_{j}+\nu_{j}-\mu_{j}+\sigma_{j}-\eta_{j}+1}{p_{j}}\right)} \\
& \times \frac{\Gamma\left(\sum_{j=1}^{n} \frac{\alpha_{j}+1}{p_{j}}+1\right)}{\prod_{j=1}^{s} \Gamma\left(\sum_{t=\widehat{k}_{j-1}+1}^{\widehat{k}_{j}} \frac{\alpha_{t}+\sigma_{t}+1}{p_{t}}\right)} \times z^{\alpha+\nu-\mu+\sigma-\eta}
\end{aligned}
$$

Similarly, we also have

$$
T_{a \xi^{\nu} \bar{\xi}^{\mu}} T_{b \xi^{\sigma} \bar{\xi}^{\eta}} z^{\alpha}=
$$




$$
\begin{aligned}
& \int_{\Delta_{m}^{n}} b\left(r_{1}, \ldots, r_{n}\right) \prod_{j=1}^{s} r_{j}^{\left(\sum_{t=\widehat{k}_{j-1}+1}^{\widehat{k}_{j}} \frac{2 \alpha_{t}+2}{p_{t}}-1\right)} d r_{j} \\
& \times \frac{4^{s} \prod_{t=1}^{n} \Gamma\left(\frac{\alpha_{t}+\sigma_{t}+1}{p_{t}}\right) \prod_{j=1}^{s}\left(\sum_{t=\widehat{k}_{j-1}+1}^{\widehat{k}_{j}} \frac{1}{p_{t}}\right) \Gamma\left(\sum_{j=1}^{n} \frac{\alpha_{j}+1}{p_{j}}+1\right)}{\prod_{j=1}^{n} \Gamma\left(\frac{\alpha_{j}+\sigma_{j}-\eta_{j}+1}{p_{j}}\right) \prod_{j=1}^{s} \Gamma\left(\sum_{t=\widehat{k}_{j-1}+1}^{\widehat{k}_{j}} \frac{\alpha_{t}+\sigma_{t}+1}{p_{t}}\right)} \\
& \times \int_{\Delta_{m}^{n}} a\left(r_{1}, \ldots, r_{n}\right) \prod_{j=1}^{s} r_{j}^{\left(\sum_{t=\widehat{k}_{j-1}+1}^{\widehat{k}_{j}} \frac{2 \alpha_{t}+2}{p_{t}}-1\right)} d r_{j} \\
& \times \frac{4^{s} \prod_{t=1}^{n} \Gamma\left(\frac{\alpha_{t}+\sigma_{t}-\eta_{t}+\nu_{t}+1}{p_{t}}\right) \prod_{j=1}^{s}\left(\hat{k}_{t=\widehat{k}_{j-1}+1} \frac{1}{p_{t}}\right)}{\prod_{j=1}^{n} \Gamma\left(\frac{\alpha_{j}+\sigma_{j}-\eta_{j}+\nu_{j}-\mu_{j}+1}{p_{j}}\right)} \\
& \times \frac{\Gamma\left(\sum_{j=1}^{n} \frac{\alpha_{j}+1}{p_{j}}+1\right)}{\prod_{j=1}^{s} \Gamma\left(\sum_{t=\widehat{k}_{j-1}+1}^{\widehat{k}_{j}} \frac{\alpha_{t}+\nu_{t}+1}{p_{t}}\right)} \times z^{\alpha+\nu-\mu+\sigma-\eta}
\end{aligned}
$$

Therefore, we conclude that $T_{a \xi^{\nu}} \bar{\xi}^{\mu} T_{b \xi^{\sigma} \bar{\xi}^{\eta}} z^{\alpha}=T_{b \xi^{\sigma} \bar{\xi}^{\eta}} T_{a \xi^{\nu} \bar{\xi}^{\mu}}$ if and only if

$$
\prod_{t=1}^{n} \frac{\Gamma\left(\frac{\alpha_{t}+\nu_{t}+1}{p_{t}}\right) \Gamma\left(\frac{\alpha_{t}+\nu_{t}-\mu_{t}+\sigma_{t}+1}{p_{t}}\right)}{\Gamma\left(\frac{\alpha_{t}+\nu_{t}-\mu_{t}+1}{p_{t}}\right)}=\prod_{t=1}^{n} \frac{\Gamma\left(\frac{\alpha_{t}+\sigma_{t}+1}{p_{t}}\right) \Gamma\left(\frac{\alpha_{t}+\sigma_{t}-\eta_{t}+\nu_{t}+1}{p_{t}}\right)}{\Gamma\left(\frac{\alpha_{t}+\sigma_{t}-\eta_{t}+1}{p_{t}}\right)} .
$$

Finally, one can easily check that the latter identity holds for every $\alpha \in \mathbb{N}^{n}$ if and only if the conclusion of the statement holds. This proves the Theorem.

The above implies one of our main results: the construction of a commutative Banach algebra of Toeplitz operators on the domain $\Omega_{p}^{n}$. Note that the rest of this section generalizes the results found in [12].

Definition 4.3. Let $k \in \mathbb{Z}_{+}^{s}$ be a partition of $n$ and $h \in \mathbb{Z}_{+}^{s}$ be such that $1 \leq h_{j} \leq$ $k_{j}-1$ for all $j=1, \ldots s$. Denote by $\mathcal{A}_{k, h}\left(\Omega_{p}^{n}\right)$ the set of symbols $\varphi \in \mathcal{H} \mathcal{R}_{k}\left(\Omega_{p}^{n}\right)$ that satisfy the following properties.

(1) The symbol is of the form $\varphi=a \xi^{\nu} \bar{\xi}^{\mu}$ where $a \in \mathcal{R}_{k}\left(\Omega_{p}^{n}\right)$ and $\nu, \mu \in \mathbb{N}^{n}$ so that $\xi^{\nu} \bar{\xi}^{\mu} \in \mathcal{H}_{k}\left(\Omega_{p}^{n}\right)$.

(2) The multi-indices $\nu, \mu$ are orthogonal and satisfy

$$
\sum_{t=\widehat{k}_{j-1}+1}^{\widehat{k}_{j}} \frac{\nu_{t}-\mu_{t}}{p_{t}}=\sum_{t=1}^{k_{j}} \frac{\left(\nu_{(j)}\right)_{t}-\left(\mu_{(j)}\right)_{t}}{\left(p_{(j)}\right)_{t}}=0
$$

for all $j=1, \ldots, s$. 
(3) The multi-indices $\nu, \mu$ satisfy

$$
\begin{aligned}
& \qquad \nu_{\widehat{k}_{j}+t_{1}}=\left(\nu_{(j)}\right)_{t_{1}}=0, \quad \mu_{\widehat{k}_{j}+t_{2}}=\left(\mu_{(j)}\right)_{t_{2}}=0 \\
& \text { for all } 1 \leq t_{2} \leq h_{j}<t_{1} \leq k_{j} \text { and } j=1, \ldots, s \text {. }
\end{aligned}
$$

Remark 4.4. From now on, for $p$ as before we will denote by $\operatorname{lcm}(p)$ the least common multiple of $p_{1}, \ldots, p_{n}$. Also, let us denote

$$
\Lambda(p)=\operatorname{lcm}(p)\left(\frac{1}{p_{1}}, \ldots, \frac{1}{p_{n}}\right),
$$

which belongs to $\mathbb{Z}_{+}^{n}$. With these conventions, multiplying by $\operatorname{lcm}(p)$ shows that condition (2) from Definition 4.3 is equivalent to

2' The multi-indices $\nu, \mu$ are orthogonal and satisfy

$$
\Lambda(p)_{(j)} \cdot\left(\nu_{(j)}-\mu_{(j)}\right)=\sum_{t=\widehat{k}_{j-1}+1}^{\widehat{k}_{j}} \Lambda(p)_{t}\left(\nu_{t}-\mu_{t}\right)=0,
$$

for every $j=1, \ldots, s$.

On the other hand, we observe that the set of symbols $\mathcal{A}_{k, h}\left(\Omega_{p}^{n}\right)$ reduces to those considered in [12] when $p=(1, \ldots, 1)$ for which we have $\Omega_{p}^{n}=\mathbb{B}^{n}$.

As an immediate consequence of Definition 4.3 and Theorem 4.2 we obtain the following result.

Theorem 4.5 (Commutative Banach algebra in $\left.\mathcal{A}^{2}\left(\Omega_{p}^{n}\right)\right)$. Let $k \in \mathbb{Z}_{+}^{s}$ be a partition of $n$ and $h \in \mathbb{Z}_{+}^{s}$ be such that $1 \leq h_{j} \leq k_{j}-1$ for all $j=1, \ldots s$. Then, the Banach algebra of Toeplitz operators generated by the symbols in $\mathcal{A}_{k, h}\left(\Omega_{p}^{n}\right)$ is commutative in the weighted Bergman space $\mathcal{A}^{2}\left(\Omega_{p}^{n}\right)$.

\section{Bundles of LAGRANGIAN FRAMES AND QUASI-HOMOGENEOUS SYMBOLS}

We will provide a geometric construction associated to the set of symbols $\mathcal{A}_{k, h}\left(\Omega_{p}^{n}\right)$ introduced in the previous section. In particular, we will consider fixed $k \in \mathbb{Z}_{+}^{s}$ a partition of $n$ and $h \in \mathbb{Z}_{+}^{s}$ satisfying the conditions of Definition 4.3 . We will also use the notation introduced in Remark 4.4 as well as the equivalence between condition (2') from such remark and condition (2) from Definition 4.3.

We recall that $\Omega_{p}^{n}$ is a Reinhardt domain and so the action

$$
\begin{aligned}
\mathbb{T}^{n} \times \Omega_{p}^{n} & \rightarrow \Omega_{p}^{n} \\
(\tau, z) & \mapsto\left(\tau_{1} z_{1}, \ldots, \tau_{n} z_{n}\right)
\end{aligned}
$$

is holomorphic. Hence, the action is isometric for the Kähler metric defined by the Bergman kernel of $\Omega_{p}^{n}$.

As noted in [8], the known commutative $C^{*}$-algebras of Toeplitz operators have an associated Abelian group of isometries. We refer to [3, [7, [10] and [1] for a detailed discussion of such behavior. We have shown that this property extends to quasi-homogeneous symbols on the complex projective space $\mathbb{P}^{n}(\mathbb{C})$ in a suitable fashion. To describe the corresponding behavior for $\Omega_{p}^{n}$ we consider the following homomorphism built from our current information.

$$
\begin{aligned}
\pi_{p}: \mathbb{T}^{n} & \rightarrow \mathbb{T}^{n} \\
t & \mapsto t^{\Lambda(p)}=\left(t_{1}^{\Lambda(p)_{1}}, \ldots, t_{n}^{\Lambda(p)_{n}}\right) .
\end{aligned}
$$


We observe that $\pi_{p}$ is a surjective homomorphism of Lie groups. Moreover, $\pi_{p}$ is a local diffeomorphism. This homomorphism allows us to give a simpler description of the group associated to the symbols that we have considered.

Theorem 5.1 (Abelian group associated to $\mathcal{A}_{k, h}\left(\Omega_{p}^{n}\right)$ ). Let $k \in \mathbb{Z}_{+}^{s}$ be a partition of $n$ and $h \in \mathbb{Z}_{+}^{s}$ be such that the conditions of Definition 4.3 are satisfied. Let $\mathcal{T}_{k}(p)$ be the maximal connected subgroup of $\mathbb{T}^{n}$ that satisfies

- $\tau \in \mathcal{T}_{k}(p)$ if and only if $\varphi(\tau z)=\varphi(z)$ for every $\varphi \in \mathcal{A}_{k, h}\left(\Omega_{p}^{n}\right)$ and $z \in \Omega_{p}^{n}$. Then, $\mathcal{T}_{k}(p)=\pi_{p}\left(\mathbb{T}_{k}^{s}\right)$, where $\mathbb{T}_{k}^{s}$ is the subgroup of $\mathbb{T}^{n}$ given by

$$
\mathbb{T}_{k}^{s}=\left\{\tau \in \mathbb{T}^{n}: \exists \omega \in \mathbb{T}^{s} \text { such that } \tau_{(j)}=\left(\omega_{j}, \ldots, \omega_{j}\right) \forall j=1, \ldots, s\right\} .
$$

In particular, $\mathcal{T}_{k}(p)$ is a closed subgroup of $\mathbb{T}^{n}$ isomorphic to $\mathbb{T}^{s}$.

Proof. Since $\pi_{p}$ is surjective, we can consider the elements of $\mathbb{T}^{n}$ to be given in the form $\pi_{p}(\tau)$ for $\tau \in \mathbb{T}^{n}$.

Let $\varphi \in \mathcal{A}_{k, h}\left(\Omega_{p}^{n}\right)$ be given as in Definition 4.3, in the form $\varphi=a \xi^{\nu} \bar{\xi}^{\mu}$. In coordinates this can be written as

$$
\varphi(z)=a\left(\left|z_{(1)}\right|, \ldots,\left|z_{(s)}\right|\right) \prod_{j=1}^{s}\left(\frac{z_{(j)}}{\left|z_{(j)}\right|}\right)^{\nu_{(j)}}\left(\frac{\bar{z}_{(j)}}{\left|z_{(j)}\right|}\right)^{\mu_{(j)}},
$$

which yields for every $\pi_{p}(\tau) \in \mathbb{T}^{n}$

$$
\begin{aligned}
& \varphi\left(\pi_{p}(\tau) z\right)= \\
& \left.a\left(\left|z_{(1)}\right|, \ldots,\left|z_{(s)}\right|\right) \prod_{j=1}^{s} \pi_{p}(\tau)_{(j)}^{\nu_{(j)}} \overline{\pi_{p}(\tau)}\right)_{(j)}^{\mu_{(j)}} \prod_{j=1}^{s}\left(\frac{z_{(j)}}{\left|z_{(j)}\right|}\right)^{\nu_{(j)}}\left(\frac{\bar{z}_{(j)}}{\left|z_{(j)}\right|}\right)^{\mu_{(j)}} .
\end{aligned}
$$

We conclude that $\pi_{p}(\tau) \in \mathcal{T}_{k}(p)$ if and only if

$$
\prod_{j=1}^{s}\left(\tau_{(j)}^{\Lambda(p)_{(j)}}\right)^{\nu_{(j)}-\mu_{(j)}}=\prod_{j=1}^{s} \pi_{p}(\tau)_{(j)}^{\nu_{(j)}} \bar{\pi}_{p(\tau)}^{\mu_{(j)}}=1,
$$

for every $\nu, \mu \in \mathbb{Z}_{+}^{n}$ that satisfies conditions (2) and (3) from Definition 4.3. We have used the fact that $\pi_{p}(\tau)_{(j)}=\tau_{(j)}^{\Lambda(p)_{(j)}}$ for every $j=1, \ldots, s$.

For $\tau \in \mathbb{T}_{k}^{s}$, let $\omega \in \mathbb{T}^{s}$ such that $\tau_{(j)}=\left(\omega_{j}, \ldots, \omega_{j}\right)$ for all $j$. Then, we have

$$
\left(\tau_{(j)}^{\Lambda(p)_{(j)}}\right)^{\nu_{(j)}-\mu_{(j)}}=\omega^{\Lambda(p)_{(j)} \cdot\left(\nu_{(j)}-\mu_{(j)}\right)}=1
$$

which vanishes for all $j$ since condition $\left(2^{\prime}\right)$ holds. Since $\mathbb{T}_{k}^{s}$ is connected, this shows that $\pi_{p}\left(\mathbb{T}_{k}^{s}\right) \subset \mathcal{T}_{k}(p)$. By the connectedness of $\mathcal{T}_{k}(p)$ and since $\pi_{p}$ is a local diffeomorphism, to conclude that $\pi_{p}\left(\mathbb{T}_{k}^{s}\right)=\mathcal{T}_{k}(p)$ it is enough to show that $\operatorname{dim}\left(\mathcal{T}_{k}(p)\right)=s$. Notice that the inclusion $\pi_{p}\left(\mathbb{T}_{k}^{s}\right)=\mathcal{T}_{k}(p)$ already shows that $s \leq \operatorname{dim}\left(\mathcal{T}_{k}(p)\right)$.

Let $\pi_{p}(\tau) \in \mathcal{T}_{k}(p)$ be given, and choose $j_{0} \in\{1, \ldots, s\}$ and $t_{1}, t_{2} \in \mathbb{Z}^{+}$such that $1 \leq t_{1} \leq h_{j_{0}}<t_{2} \leq k_{j_{0}}$. For such data let us define

$$
\begin{aligned}
& \left(\nu_{(j)}\right)_{t}= \begin{cases}\left(p_{\left(j_{0}\right)}\right)_{t_{1}} & \text { if } j=j_{0}, t=t_{1} \\
0 & \text { otherwise }\end{cases} \\
& \left(\mu_{(j)}\right)_{t}= \begin{cases}\left(p_{\left(j_{0}\right)}\right)_{t_{2}} & \text { if } j=j_{0}, t=t_{2} \\
0 & \text { otherwise }\end{cases}
\end{aligned}
$$


Then, it is easily seen that $\nu, \mu$ satisfy the conditions from Definition 4.3. This implies that (5.1) is satisfied for our current $\tau$ and such multi-indices $\nu, \mu$. Moreover, (5.1) reduces to the identity

$$
\begin{aligned}
\left.\left(\tau_{\left(j_{0}\right)}\right)\right)_{t_{1}}^{\operatorname{lcm}(p)}\left(\tau_{\left(j_{0}\right)}\right)_{t_{2}}^{-\operatorname{lcm}(p)}= & \\
& \left(\tau_{\left(j_{0}\right)}\right)_{t_{1}}^{\left.\left.(\Lambda(p))_{\left(j_{0}\right)}\right) t_{1}\left(p_{\left(j_{0}\right)}\right)\right)_{t_{1}}}\left(\tau_{\left(j_{0}\right)}\right)_{t_{2}}^{\left.-(\Lambda(p))_{\left(j_{0}\right)}\right) t_{2}\left(p_{\left(j_{0}\right)}\right) t_{2}}=1 .
\end{aligned}
$$

Hence, given the choices of $j_{0}, t_{1}, t_{2}$, we conclude that there exists $\omega \in \mathbb{T}^{s}$ such that

$$
\left(\tau_{(j)}\right)_{t}^{\operatorname{lcm}(p)}=\omega_{j}
$$

for every $j=1, \ldots, s$ and $t \in\left\{1, \ldots, k_{j}\right\}$. If we consider the homomorphism

$$
\begin{aligned}
\rho: \mathbb{T}^{n} & \rightarrow \mathbb{T}^{n} \\
\tau & \mapsto\left(\tau_{1}^{\mathrm{lcm}(p)}, \ldots, \tau_{n}^{\mathrm{lcm}(p)}\right),
\end{aligned}
$$

then we have just proved that $\rho\left(\mathcal{T}_{k}(p)\right) \subset \mathbb{T}_{k}^{s}$. We note that $\rho$ is a local diffeomorphism and so we conclude that $\operatorname{dim}\left(\mathcal{T}_{k}(p)\right) \leq s$. This proves that $\operatorname{dim}\left(\mathcal{T}_{k}(p)\right)=s$ and, as remarked above, it shows that $\mathcal{T}_{k}(p)=\pi_{p}\left(\mathbb{T}_{k}^{s}\right)$.

For the last claim, observe that since $\mathbb{T}_{k}^{s} \simeq \mathbb{T}^{s}$ the group $\mathcal{T}_{k}(p)=\pi_{p}\left(\mathbb{T}_{k}^{s}\right)$ is compact and thus closed in $\mathbb{T}^{n}$. Furthermore, the fact that $\pi_{p}$ is a local isomorphism implies that $\mathcal{T}_{k}(p) \simeq \mathbb{T}_{k}^{s} \simeq \mathbb{T}^{s}$ since all the groups involved are compact and Abelian.

Remark 5.2. We note that the group $\mathcal{T}_{k}(p)$ does not depend on $h$. For $p_{0}=$ $(1, \ldots, 1)$ the domain under consideration is the unit ball $\Omega_{(1, \ldots, 1)}^{n}=\mathbb{B}^{n}$, and in this case $\Lambda\left(p_{0}\right)=(1, \ldots, 1)$ as well. Hence, $\pi_{p_{0}}=i d_{\mathbb{T}^{n}}$ is the identity homomorphism of $\mathbb{T}^{n}$ and so $\mathcal{T}_{k}\left(p_{0}\right)=\mathbb{T}_{k}^{s}$.

Following the references mentioned above, we now proceed to construct Lagrangian frames associated to our symbols in $\mathcal{A}_{k, h}\left(\Omega_{p}^{n}\right)$ by using the torus $\mathcal{T}_{k}(p)$.

First, we note that the homomorphism $\pi_{p}$ defined above can be more generally considered as the homomorphism of $\mathbb{C}^{* n}$ with the same expression

$$
\begin{aligned}
\pi_{p}: \mathbb{C}^{* n} & \rightarrow \mathbb{C}^{* n} \\
\zeta & \mapsto\left(\zeta_{1}^{\Lambda(p)_{1}}, \ldots, \zeta_{n}^{\Lambda(p)_{n}}\right) .
\end{aligned}
$$

We thus introduce the complexification of $\mathcal{T}_{k}(p)$ as given by

$$
\mathcal{T}_{k}^{\mathbb{C}}(p)=\pi_{p}\left(\mathbb{C}_{k}^{* s}\right)
$$

for the following subgroup of $\mathbb{C}^{* n}$

$$
\mathbb{C}_{k}^{* s}=\left\{\zeta \in \mathbb{C}^{* n}: \exists \omega \in \mathbb{C}^{* s} \text { such that } \zeta_{(j)}=\left(\omega_{j}, \ldots, \omega_{j}\right) \forall j=1, \ldots, s\right\} .
$$

For the corresponding construction in the case of the projective space we considered an action of the complexified group. However, in this case, we have only a local action of $\mathcal{T}_{k}^{\mathbb{C}}(p)$ on $\Omega_{p}^{n}$ given by the expression

$$
\left(\pi_{p}(\zeta), z\right) \mapsto \pi_{p}(\zeta) z=\left(\zeta_{1}^{\Lambda(p)_{1}} z_{1}, \ldots, \zeta_{n}^{\Lambda(p)_{n}} z_{n}\right) .
$$

where $\left(\pi_{p}(\zeta), z\right) \in \mathcal{T}_{k}^{\mathbb{C}}(p) \times \Omega_{p}^{n}$ is restricted to those pairs such that $\pi_{p}(\zeta) z \in \Omega_{p}^{n}$. More precisely, we have the following easy to prove result. It also shows that the local action of $\mathcal{T}_{k}^{\mathbb{C}}(p)$ extends the action of $\mathcal{T}_{k}(p)$. 
Lemma 5.3. With the above notation, the subset of pairs $\left(\pi_{p}(\zeta), z\right) \in \mathcal{T}_{k}^{\mathbb{C}}(p) \times \Omega_{p}^{n}$ such that $\pi_{p}(\zeta) z \in \Omega_{p}^{n}$ is an open subset of $\mathcal{T}_{k}^{\mathbb{C}}(p) \times \Omega_{p}^{n}$. Such open set contains

$$
\pi_{p}\left(\mathbb{B}_{0} \times \cdots \times \mathbb{B}_{0}\right) \times \Omega_{p}^{n},
$$

where the first factor is the image under $\pi_{p}$ of the product of $n$ copies of $\mathbb{B}_{0}$, the punctured disc $\mathbb{B} \backslash\{0\}$ in $\mathbb{C}$. In particular, the local action is defined on $\mathcal{T}_{k}(p) \times \Omega_{p}^{n}$ where it is given by the action of $\mathcal{T}_{k}(p)$ on $\Omega_{p}^{n}$ considered above.

We observe that the local action of $\mathcal{T}_{k}^{\mathbb{C}}(p)$ is locally holomorphic in the sense that for $\zeta \in \mathcal{T}_{k}^{\mathbb{C}}(p)$ fixed the map defined by (5.2) is locally holomorphic.

To perform our geometric constructions it is better to work with a global action. Hence, we consider the corresponding action of $\mathcal{T}_{k}^{\mathbb{C}}(p)$ on $\mathbb{C}^{n}$ given by the same expression

$$
\begin{aligned}
\mathcal{T}_{k}^{\mathbb{C}}(p) \times \mathbb{C}^{n} & \rightarrow \mathbb{C}^{n} \\
\left(\pi_{p}(\zeta), z\right) & \mapsto \pi_{p}(\zeta) z=\left(\zeta_{1}^{\Lambda(p)_{1}} z_{1}, \ldots, \zeta_{n}^{\Lambda(p)_{n}} z_{n}\right) .
\end{aligned}
$$

It is easy to see that the orbits for the action of $\mathcal{T}_{k}^{\mathbb{C}}(p)$ on $\mathbb{C}^{n}$ have varying dimensions, so we introduce the following stratification that collects (some) orbits with the same dimension. For every $j=1, \ldots, s$ we let

$$
V_{j}=\left\{z \in \mathbb{C}^{n}: z_{(1)}=0, \ldots, z_{(j-1)}=0, \text { and } z_{(j)} \neq 0, \ldots, z_{(s)} \neq 0\right\} .
$$

It is easy to see that, for every $j$, the set $V_{j}$ is a complex submanifold of $\mathbb{C}^{n}$ that is invariant under the action of $\mathcal{T}_{k}^{\mathbb{C}}(p)$. Recall that an action is free when its stabilizers are trivial. One can consider free actions by the introduction of the following subgroups of $\mathcal{T}_{k}^{\mathbb{C}}(p)$. We let for every $j=1, \ldots, s$

$$
G_{j}=\pi_{p}\left(\left\{\zeta \in \mathbb{C}_{k}^{* s}: \zeta_{t}=1 \text {, for all } j=1, \ldots, \widehat{k}_{j-1}\right\}\right),
$$

which defines a descending collection of groups with $G_{1}=\mathcal{T}_{k}^{\mathbb{C}}(p)$. Also note that $G_{j} \simeq \mathbb{C}^{*(s+1-j)}$ for all $j$. Consider the subgroups of $\mathcal{T}_{k}^{\mathbb{C}}(p)$ given by

$$
\mathcal{T}_{j}=G_{j} \cap \mathcal{T}_{k}(p),
$$

for every $j=1, \ldots, s$. Hence, the subgroups $\mathcal{T}_{j}$ form a descending collection of compact subgroups of $\mathcal{T}_{k}(p)$ such that $\mathcal{T}_{1}=\mathcal{T}_{k}(p)$ and satisfying $\mathcal{T}_{j} \simeq \mathbb{T}^{s+1-j}$.

The following result is an easy consequence of the definitions and the fact that the equations $z_{(1)}=0, \ldots, z_{(j-1)}=0$ define closed complex submanifolds of $\mathbb{C}^{n}$. Note that the set $V_{1} \cup \cdots \cup V_{s}$ is an open conull dense subset of $\mathbb{C}^{n}$.

Lemma 5.4. For the partition $V_{1} \cup \cdots \cup V_{s} \subset \mathbb{C}^{n}$ and the groups $G_{1}, \ldots, G_{s}$ defined above, the following properties hold for every $j=1, \ldots, s$.

(1) The subset $V_{j} \cup \cdots \cup V_{s}$ is a closed complex submanifold of $\mathbb{C}^{n}$.

(2) The submanifold $V_{j}$ is open in $V_{j} \cup \cdots \cup V_{s}$.

(3) The action of the group $G_{j}$ on $\mathbb{C}^{n}$ leaves invariant $V_{j} \cup \cdots \cup V_{s}$.

(4) The submanifold $V_{j}$ is the largest subset of $V_{j} \cup \cdots \cup V_{s}$ where the local action of $G_{j}$ is free.

Next we obtain a collection of fiber bundles whose total spaces are the submanifolds $V_{j}$ and whose structure groups are the corresponding $G_{j}$. We refer to [5] for more details on the notions related to fiber bundles. The following result thus provides the first geometric structure associated to the symbols in $\mathcal{A}_{k, h}\left(\Omega_{p}^{n}\right)$. 
Theorem 5.5 (Principal bundles associated to $\mathcal{A}_{k, h}\left(\Omega_{p}^{n}\right)$ ). Let $k \in \mathbb{Z}_{+}^{s}$ be a partition of $n$ and $h \in \mathbb{Z}_{+}^{s}$ be such that the conditions of Definition 4.3 are satisfied. Consider the submanifolds $V_{1}, \ldots, V_{s}$ of $\mathbb{C}^{n}$ and the subgroups $G_{1}, \ldots, G_{s}$ of $\mathcal{T}_{k}^{\mathbb{C}}(p)$ defined above. Then, the following property is satisfied for every $j=1, \ldots, s$.

- The quotient space $G_{j} \backslash V_{j}$ is a complex manifold so that the natural quotient map $V_{j} \rightarrow G_{j} \backslash V_{j}$ is a complex fiber bundle with structure group $G_{j} \simeq$ $\mathbb{C}^{*(s+1-j)}$. In particular, every $G_{j}$-orbit is a complex submanifold of $\mathbb{C}^{n}$.

Proof. By Lemma 5.3 the action of $G_{j}$ on $V_{j}$ is free and so by the results from [2] it is enough to show that the action of $G_{j}$ is proper. Recall that a $G$-action on a manifold $V$ is proper if for every compact subset $K \subset V$ the set

$$
\{g \in G: g K \cap K \neq \emptyset\}
$$

has compact closure in $G$.

Let $K \subset V_{j}$ be a compact subset. From the definition of $V_{j}$ it follows we have

- if $z \in K$, then $z_{(t)} \neq 0$ for every $t=j, \ldots, s$,

- there exists $c_{1}, c_{2}>0$ such that $c_{1} \leq\left|z_{t}\right| \leq c_{2}$, for all $t=\widehat{k}_{(j-1)}+1, \ldots, n$, and for every $z \in K$.

Let $\pi_{p}(\zeta) \in G_{j}$ be given, so that in particular we have $\zeta_{t}=1$ for all $t=1, \ldots, \widehat{k}_{(j-1)}$. If $\pi_{p}(\zeta) K \cap K \neq \emptyset$, then there exists $z, z^{\prime} \in K$ such that

$$
\zeta_{t}^{\Lambda(p)_{t}} z_{t}=z_{t}^{\prime}
$$

for all $t=\widehat{k}_{(j-1)}+1, \ldots, n$. This implies that

$$
C^{-1} \leq\left|\zeta_{t}\right|^{\Lambda(p)_{t}} \leq C
$$

for every $t=\widehat{k}_{(j-1)}+1, \ldots, n$, if we take $C=c_{2} / c_{1}$. Since this defines a condition with compact closure in $G_{j}$, we conclude that the $G_{j}$-action on $V_{j}$ is compact.

The structure considered in the previous result is easily seen to induced a corresponding one on the domain $\Omega_{p}^{n}$. Its proof is an easy consequence of Lemma 5.4 and Theorem 5.5 since $\Omega_{p}^{n}$ is open in $\mathbb{C}^{n}$.

Theorem 5.6. Let $k \in \mathbb{Z}_{+}^{s}$ be a partition of $n$ and $h \in \mathbb{Z}_{+}^{s}$ be such that the conditions of Definition 4.3 are satisfied. Consider the submanifolds $V_{1}, \ldots, V_{s}$ of $\mathbb{C}^{n}$ and the subgroups $G_{1}, \ldots, G_{s}$ of $\mathcal{T}_{K}^{\mathbb{C}}(p)$ defined above, and denote $\widehat{V}_{j}=V_{j} \cap \Omega_{p}^{n}$ for every $j=1, \ldots, s$. Then, the following properties are satisfied for every $j=$ $1, \ldots, s$

(1) The subset $\widehat{V}_{j} \cup \widehat{V}_{s}$ is a closed complex submanifold of $\Omega_{p}^{n}$.

(2) The submanifold $\widehat{V}_{j}$ is open in $\widehat{V}_{j} \cup \widehat{V}_{s}$.

(3) The intersection of the $G_{j}$-orbits in $V_{j}$ with $\Omega_{p}^{n}$ defines a foliation $\mathfrak{F}_{j}$ by complex submanifolds in $\widehat{V}_{j}$.

We now introduce the notion of a Lagrangian frame that was first considered in 9], and refer to this work for the details of the geometric notions involved.

Definition 5.7. For a Kähler manifold $N$, a Lagrangian frame is a pair of smooth foliations $(\mathfrak{F}, \mathcal{O})$ that satisfy the following properties

(1) Both foliations are Lagrangia. In other words, their leaves are Lagrangian submanifolds of $N$. 
(2) If $L_{1}$ and $L_{2}$ are leaves of $\mathfrak{F}$ and $\mathcal{O}$, respectively, then $T_{x} L_{1} \perp T_{x} L_{2}$ at every point $x \in L_{1} \cap L_{2}$.

(3) The foliation $\mathcal{O}$ is Riemannian. In other words, the the Riemannian metric of $N$ is invariant under the leaf holonomy of $\mathcal{O}$.

(4) The foliation $\mathfrak{F}$ is totally geodesic. In other words, the leaves of $\mathfrak{F}$ are totally geodesic submanifolds of $N$.

And in this case, we refer to $\mathcal{O}$ and $\mathfrak{F}$ as the Riemannian and totally geodesic foliations, respectively, of the Lagrangian frame.

It is a remarkable fact that the symbols in $\mathcal{A}_{k, h}\left(\Omega_{p}^{n}\right)$ yield a Lagrangian frame on $\Omega_{p}^{n}$, as the following result establishes.

Theorem 5.8 (Lagrangian frames associated to $\mathcal{A}_{k, h}\left(\Omega_{p}^{n}\right)$ ). Let $k \in \mathbb{Z}_{+}^{s}$ be a partition of $n$ and $h \in \mathbb{Z}_{+}^{s}$ be such that the conditions of Definition 4.3 are satisfied. Then, for every $j=1, \ldots, s$ and for every leaf $L$ in the foliation $\mathfrak{F}_{j}$ of $\widehat{V}_{j}$ the following properties hold.

(1) The action of $\mathcal{T}_{j}$ restricted to $L$ defines a Riemannian foliation $\mathcal{O}_{L}$ on whose leaves every symbol in $\mathcal{A}_{k, h}\left(\Omega_{p}^{n}\right)$ is constant.

(2) The vector bundle $T \mathcal{O}_{L}^{\perp}$ defined as the orthogonal complement of $T \mathcal{O}_{L}$ in $T L$ is integrable to a totally geodesic foliation $\mathcal{J O}_{L}$.

(3) The pair $\left(\mathcal{O}_{L}, \mathcal{J O}_{L}\right)$ is a Lagrangian frame in $L$ for the Kähler structure inherited from $\Omega_{P}^{n}$.

Proof. Note that $L$ is an open set of a $G_{j}$-orbit in $V_{j}$. Hence, the complex dimension of $L$ is $\operatorname{dim}_{\mathbb{C}} G_{j}=s+1-j$. Hence, the real dimension of the $\mathcal{T}_{j}$-orbits in $L$ is $\operatorname{dim} \mathcal{T}_{j}=s+1-j$. Also, $\mathcal{T}_{j}$ leaves invariant both $V_{j}$ and $\Omega_{p}^{n}$, and so it leaves invariant $L$. Since the $\mathcal{T}_{j}$-action on $V_{j}$ is free, it is so on $L$ and hence it defines a foliation $\mathcal{O}_{L}$ in $L$.

Since $\mathcal{T}_{j} \subset \mathbb{T}^{n}$ which acts by isometries on the Reinhardt domain $\Omega_{p}^{n}$, it follows that the foliation $\mathcal{O}_{L}$ is Riemannian in $L$ for the Kähler structure on $L$ inherited from $\Omega_{p}^{n}$. We refer to [9] for the relevant results. We also know from [9] that the $\mathbb{T}^{n}$-orbits on $\Omega_{p}^{n}$ are Lagrangian. Since $\mathcal{T}_{j}$ acts on $L$ by a restriction of the $\mathbb{T}^{n}$ action, it follows that the $\mathcal{T}_{j}$-orbits are isotropic in $L$; here we have also used that $L$ has the Kähler structure inherited from that of $\Omega_{p}^{n}$. Since the real dimension of the $\mathcal{T}_{j}$-orbits in $L$ and the complex dimension of $L$ are the same, we conclude that $\mathcal{O}_{L}$ is a Lagrangian foliation. Also observe that, by Theorem [5.1] the symbols in $\mathcal{A}_{k, h}\left(\Omega_{p}^{n}\right)$ are constant on the leaves of the foliation $\mathcal{O}_{L}$.

Since the orthogonal complement to the tangent bundle of a Riemannian foliation is totally geodesic (see [9]), to complete the proof it is enough to show that $T \mathcal{O}_{L}^{\perp}=$ $i T \mathcal{O}_{L}$ is an integrable vector subbundle of $T L$.

To prove the integrability of $i T \mathcal{O}_{F}$ let us consider $\left\{X_{t}: t=1, \ldots, s+1-j\right\}$ a base for the Lie algebra of $\mathcal{T}_{j}$. The $\mathcal{T}_{j}$-action on $L$ induces a family of vector fields $\left\{X_{t}^{*}: t=1, \ldots, s+1-j\right\}$ on $L$ characterized as those having flows given by $\left\{\exp \left(X_{t}\right): t=1, \ldots, s+1-j\right\}$, respectively. We refer to [4] for further details on this construction. Since the $\mathcal{T}_{j}$-action on $L$ is free, it follows that the vector fields $\left\{X_{t}^{*}: t=1, \ldots, s+1-j\right\}$ are linearly independent at every point of $L$, thus defining a generating set for $T \mathcal{O}_{L}$ at every point of $L$. Furthermore, since $\mathcal{T}_{j}$ is Abelian $\left[X_{t_{1}}^{*}, X_{t_{2}}^{*}\right]=0$ for every $t_{1}, t_{2}$.

On the other hand, for $J$ the complex structure of $\Omega_{p}^{n}$, the set of vector fields $\left\{J X_{t}^{*}: t=1, \ldots, s+1-j\right\}$ yields a generating set for $i T \mathcal{O}_{F}$ at every point of $F$. 
We claim that for every $t$, the vector fields $X_{t}^{*}$ and $J X_{t}^{*}$ are holomorphic, i.e. they integrate to holomorphic local flows. First note that, dy definition, the vector fields $X_{t}^{*}$ to flows which are 1-parameter subgroups of the $\mathcal{T}_{j}$-action. Since the latter is holomorphic, we conclude that the vector fields $X_{t}^{*}$ are holomorphic.

We now consider the vector fields $J X_{t}^{*}$. First we recall that $\mathcal{T}_{j} \subset \mathcal{T}_{k}(p) \subset \mathcal{T}_{k}^{\mathbb{C}}(p)$, which implies that

$$
X_{t} \in \operatorname{Lie}\left(\mathcal{T}_{j}\right) \subset \operatorname{Lie}\left(\mathcal{T}_{k}^{\mathbb{C}}(p)\right)
$$

Since the local action of $\mathcal{T}_{k}^{\mathbb{C}}(p)$ on $\Omega_{p}^{n}$ is holomorphic we conclude that

$$
J X_{t}^{*}=\left(i X_{t}\right)^{*}
$$

In particular, $J X_{t}^{*}$ is a holomorphic vector field on $\Omega_{p}^{n}$. Once this is known, we have for every $t_{1}, t_{2}$

$$
\left[J X_{t_{1}}^{*}, J X_{t_{2}}^{*}\right]=J\left[X_{t_{1}}^{*}, J X_{t_{2}}^{*}\right]=J^{2}\left[X_{t_{1}}^{*}, X_{t_{2}}^{*}\right]=0 .
$$

Here we have used in the first and second identities the fact that $J X_{t_{2}}^{*}$ and $X_{t_{1}}^{*}$, respectively, define Lie derivatives that commute with $J$; the latter is a consequence of the fact that both vector fields are holomorphic (see [6]). Thus, we have proved that the bundle $i T \mathcal{O}_{p}$ has a set of sections that generate the fibers and commute pairwise. Hence, the integrability of $i T \mathcal{O}_{p}$ follows from Frobenius Theorem.

Finally, we mention that a result similar to Theorem 5.9 from 8 can be obtained by considering a suitable complement of $\mathcal{T}_{k}(p) \operatorname{in} \mathcal{T}_{k}^{\mathbb{C}}(p)$. However, this provides only a local action on $\Omega_{p}^{n}$ and so we omit the details of such construction.

\section{REFERENCES}

[1] D. Crocker and I. Raeburn, Toeplitz Operator on Certain weakly Pseudoconvex Domains, J. Austral. Math. Soc. Ser. A, 31 (1981), 1-14.

[2] S. Gallot, D. Hulin, J. Lafontaine, Riemannian geometry. Third edition. Universitext. Springer-Verlag, Berlin, 2004.

[3] S. Grudsky, R. Quiroga-Barranco, N. Vasilevski, Commutative $C^{*}$-algebras of Toeplitz operators and quantization on the unit disk, J. Funct. Anal. 234 (2006), no. 1, 1-44.

[4] S. Helgason, Differential geometry, Lie groups, and symmetric spaces, Pure and Applied Mathematics, 80. Academic Press, Inc., New York-London, 1978.

[5] S. Kobayashi, K. Nomizu, Foundations of differential geometry. Vol. I. Reprint of the 1969 original. Wiley Classics Library. A Wiley-Interscience Publication. John Wiley \& Sons, Inc., New York, 1996.

[6] S. Kobayashi, K. Nomizu, Foundations of differential geometry. Vol. II. Reprint of the 1969 original. Wiley Classics Library. A Wiley-Interscience Publication. John Wiley \& Sons, Inc., New York, 1996.

[7] R. Quiroga-Barranco, A. Sanchez-Nungaray, Commutative $C^{*}$-algebras of Toeplitz operators on the complex projective space, Integral Equations and Operator Theory, $\mathbf{7 1}$ (2011), no. 2, 225-243.

[8] R. Quiroga-Barranco, A. SAnchez-Nungaray, Toeplitz operators with quasi-radial quasihomogeneous symbols and bundles of Lagrangian frames, to appear in Journal of Operator Theory.

[9] R. Quiroga-Barranco, N. Vasilevski, Commutative algebras of Toeplitz operators on the Reinhardt domains, Integral Equations Operator Theory, 59 (2007), no. 1, 67-98.

[10] R. Quiroga-Barranco, N. Vasilevski, Commutative $C^{*}$-algebras of Toeplitz operators on the unit ball. I. Bargmann-type transforms and spectral representations of Toeplitz operators, Integral Equations Operator Theory, 59 (2007), no. 3, 379-419.

[11] R. Quiroga-Barranco, N. Vasilevski, Commutative $C^{*}$-algebras of Toeplitz operators on the unit ball. II. Geometry of the level sets of symbols, Integral Equations Operator Theory, 60 (2008), no. 1, 89-132. 
[12] N. VASILEvski, Quasi-radial quasi-homogeneous symbols and commutative Banach algebras of Toeplitz operators, Integral Equations Operator Theory, 66 (2010), no. 1, 141-152.

[13] K. ZHU, Spaces of Holomorphic Functions in the Unit Ball, Springer Verlag, 2005.

Centro de Investigación en Matemáticas, Guanajuato, Mexico

E-mail address: quiroga@cimat.mx

Facultad de Matemáticas, Universidad Veracruzana, Veracruz, Mexico

E-mail address: armsanchez@uv.mx 